\title{
Synthesis of 2-Diethylenetriamine Methylene-4-Nonylphenol Asphalt Emulsifier and its Investigation by Online FTIR Spectrophotometry
}

\author{
Laishun SHI ${ }^{a}$, Cunfei MA, Fang TIAN, Yang QIN, Man GUO, Qian DONG
}

School of Chemistry and Chemical Engineering, Shandong University, Jinan, 250061, China

aemail: LSHUNSH@sdu.edu.cn

Keywords: Asphalt; Emulsifier; Nonyl Phenol; Synthesis; Online FTIR

\begin{abstract}
A new cationic type asphalt emulsifier of 2-diethylenetriamine methylene-4-nonylphenol was synthesized by the reaction of nonyl phenol, diethylenetriamine and formaldehyde. The synthesis process was detected by online FTIR technique and the intermediate was found. Based upon the experimental information, a reaction mechanism was suggested for the reaction. The asphalt emulsifier has excellent surface activity and satisfactory emulsification effect. The prepared asphalt emulsion showed higher storage stability. The emulsifier belongs to rapid-set emulsifier of asphalt. It can be applied to the road pavement construction of tack coat and chip seal.
\end{abstract}

\section{Introduction}

Asphalt emulsion is a homogeneous and stable $\mathrm{O} / \mathrm{W}$ molecular system containing $55-65 \mathrm{wt} \%$ of asphalt, $35-45 \mathrm{wt} \%$ of water, and $1.0-3.0 \mathrm{wt} \%$ of emulsifiers. It has a variety of applications including industrial, agricultural and road construction. Compared with hot asphalt, the use of bituminous emulsion has the advantage of stronger adhesive power with mineral aggregate, lower energy consumption, and less pollution to the environment. Therefore, asphalt emulsifier is widely used in the sealing application which including to build and maintain road surfaces, sidewalks, runways, bicycle paths and parking lots [1-3].

According to the different speed of demulsification, asphalt emulsifier is divided into rapid-set, medium-set and slow-set types. According to the water solubility of asphalt emulsifier, it can be divided into ionic and non ionic types. Ionic types are divided into anionic types, cationic types, and zwitterionic types [4]. Anionic emulsifiers were widely used in the early stage including carboxylate, sulfonate and sulfate [5,6]. With the development of asphalt emulsifier, cationic emulsifier has become one of the most widely studied area of asphalt emulsifier. It has the advantages of excellent emulsifying property and mixing performance [7,8]. According to the chemical structure, cationic asphalt emulsifier can be divided into alkyl polyamine, quaternary ammonium salt, amide amine, imidazoline [9]. A lignin amine type asphalt emulsifier was synthesized by using the raw materials of soda lignin, secondary amines and inexpensive aminating assistants [10]. Gemini emulsifier is a new kind of surfactant, which contains more than one hydrophobic tail and hydrophilic heads in the molecules [11,12]. Dissymmetric Gemini quaternary ammonium salts cationic asphalt emulsifiers with three carbon chains were synthesized using the raw material of $\mathrm{N}, \mathrm{N}$-dodecyl dimethyl tertiary amine, N,N-octadecyl dimethyl tertiary amine, epichlorohydrin and hydrochloric acid [13].

Zwitterionic emulsifiers, including betaine and amino acid, contain both positively and negatively charged hydrophilic headgroups in the molecule [14,15]. It is effective in a wide range of $\mathrm{pH}$ value. Sulfobetaine-type zwitterionic gemini surfactants of 1,2-bis[N-methyl-N-(3-sulfopropyl)-alkylammonium]ethane were synthesized by the reaction of $\mathrm{N}, \mathrm{N}$-dimethylethylenediamine with alkyl bromide, followed by the reaction with 1,3-propane sultone [16].

In previous papers we have synthesized several types of asphalt emulsifiers $[17,18]$. In this study, a new kind of cationic asphalt emulsifier was synthesized. The synthesis process was detected by online FTIR technique. The emulsifier belongs to rapid-set emulsifier of asphalt. It can be applied to the road pavement construction of tack coat and chip seal. 


\section{Experimental}

Materials. All the reagents were of analytical grade and used without further purification. The asphalt used was A-100, which was provided by Qilu Petrochemical Company, Zibo, China. The mineral aggregate was the mixture of marble stone in different size.

Synthesis. Scheme 1 shows the synthesis route of 2-diethylenetriamine methylene-4-nonylphenol.

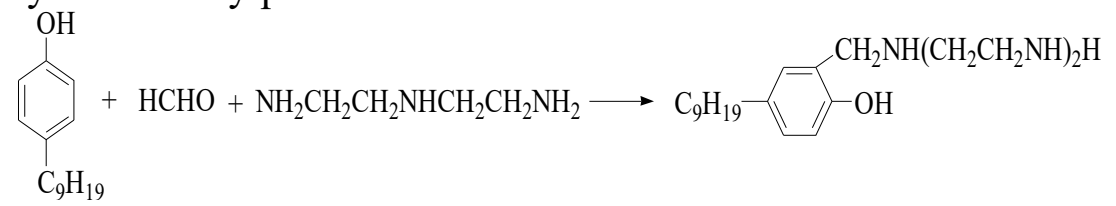

Scheme 1 Synthesis route

Nonyl phenol (16.50 g, $0.075 \mathrm{~mol})$ and diethylenetriamine $(8.51 \mathrm{~g}, 0.0825 \mathrm{~mol})$ were dissolved in $75 \mathrm{~mL}$ ethanol and stirred at $70{ }^{\circ} \mathrm{C}$ in a water bath. $37 \%$ Formaldehyde $(10.34 \mathrm{~g}, 0.1275 \mathrm{~mol})$ was added dropwise to the mixed solution. The solution was reacted at $70{ }^{\circ} \mathrm{C}$ for $6 \mathrm{~h}$.

Online FTIR analysis. The online FTIR detection was operated on a ReactIR 4000 spectrophotometer (Mettler-Toledo AutoChem, Inc., USA). FTIR spectra were recorded in the range of $650-4000 \mathrm{~cm}^{-1}$ wavenumber at a resolution of $4 \mathrm{~cm}^{-1}$. Nonyl phenol (16.50 g, $\left.0.075 \mathrm{~mol}\right)$ and diethylenetriamine $(8.51 \mathrm{~g}, 0.0825 \mathrm{~mol})$ were dissolved in $75 \mathrm{~mL}$ ethanol and stirred at $70{ }^{\circ} \mathrm{C}$ in a water bath. The detector of the online FTIR analysis apparatus was immersed in the solution during the reaction process to record the 3D online FTIR spectra. 37\% Formaldehyde (10.34 g, 0.1275mol) was added dropwise to the mixed solution in 12-17 min. The solution was reacted at $70{ }^{\circ} \mathrm{C}$ for $6 \mathrm{~h}$. The magnetic stirrer was in use during the online FTIR detection.

Preparation of asphalt emulsion. $7.5 \mathrm{~g}$ emulsifier was dissolved in $200 \mathrm{~mL}$ water. The $\mathrm{pH}$ value of the aqueous solution was adjusted to 2.0 by aqueous $30 \% \mathrm{HCl}$, and the solution was heated to $60-65^{\circ} \mathrm{C} .300 \mathrm{~g} \mathrm{~A}-100$ asphalt was heated to $120-125^{\circ} \mathrm{C}$. The asphalt emulsion was prepared by mixing the emulsifier aqueous solution and asphalt in a RHS-5 type colloid mill for $1 \mathrm{~min}$.

Mixing experiment. Mineral aggregate (300 g), $1 \mathrm{~g}$ cement and $25 \mathrm{~g}$ water were placed in a 500 $\mathrm{mL}$ bowl. Asphalt emulsion ( $40 \mathrm{~g}$ ) was then added. The mixture was mixed and agitated at a speed of 60 revolutions per minute. The mixing time was measured by observation of the mixing performance.

\section{Results and discussion}

Online FTIR Analysis. The reaction was detected by online FTIR technique. In the reaction, formaldehyde was added dropwise between 12-17 min. The reaction time was $6.0 \mathrm{~h}$. Figure 1 shows the $3 \mathrm{D}$ online infrared spectra in the whole reaction process. As can be seen, the spectra changed a lot.

Four components were found automatically during the online FTIR detection. Its FTIR spectrum was obtained (see Fig. 2-5). In the FTIR spectrum of component 1 (Fig. 2), the absorption at 1558 $\mathrm{cm}^{-1}$ (peak 1) is attributed to $\mathrm{N}-\mathrm{H}$ bending vibration. The absorption at $1458 \mathrm{~cm}^{-1}$ (peak 2) is attributed to methylene bending vibration. The absorption at $1273 \mathrm{~cm}^{-1}$ (peak 3) is attributed to C-N stretching vibration. The absorption at $1050 \mathrm{~cm}^{-1}$ (peak 4) is attributed to methylene out-of-plane swing vibration. The absorption at $879 \mathrm{~cm}^{-1}$ (peak 5) is attributed to N-H swing vibration. Therefore, the spectrum of component 1 obtained by online FTIR should be the intermediate as shown in Scheme 2.

In the FTIR spectrum of component 2 (Fig. 3), the absorptions at $1640 \mathrm{~cm}^{-1}$ (peak 1) and 1503 $\mathrm{cm}^{-1}$ (peak 2) are attributed to benzene skeleton vibration. The absorption at $1454 \mathrm{~cm}^{-1}$ (peak 3 ) is attributed to methyl and methylene bending vibration. The absorption at $1384 \mathrm{~cm}^{-1}$ (peak 4) is attributed to methyl symmetric bending vibration. The absorption at $1271 \mathrm{~cm}^{-1}$ (peak 5) is attributed to $\mathrm{O}-\mathrm{H}$ plane bending vibration. The absorptions at $1086 \mathrm{~cm}^{-1}$ (peak 6) and $1047 \mathrm{~cm}^{-1}$ (peak 7) are attributed to Ar-O stretching vibration. The absorption at $879 \mathrm{~cm}^{-1}$ (peak 8) is attributed to N-H 
plane swing vibration. The absorption at $789 \mathrm{~cm}^{-1}$ (peak 9) is attributed to methylene plane swing vibration. Therefore, the spectrum of component 2 obtained by online FTIR agrees with the target product's chemical structure.

In the FTIR spectrum of component 3 (Fig. 4), the absorption at $1717 \mathrm{~cm}^{-1}$ (peak 1) is attributed to $\mathrm{C}=\mathrm{O}$ stretching vibration of aldehyde. The absorption at $1389 \mathrm{~cm}^{-1}$ (peak 2) is attributed to $\mathrm{C}-\mathrm{H}$ bending vibration of aldehyde. The absorptions at $1086 \mathrm{~cm}^{-1}$ (peak 3) and $1047 \mathrm{~cm}^{-1}$ (peak 4) are attributed to C-H plane bending vibration. The absorptions at $879 \mathrm{~cm}^{-1}$ (peak 5) and $833 \mathrm{~cm}^{-1}$ (peak 6) are attributed to $\mathrm{C}-\mathrm{H}$ out-of-plane bending vibration. Therefore, the spectrum of component 3 obtained by online FTIR agrees with the chemical structure of formaldehyde.

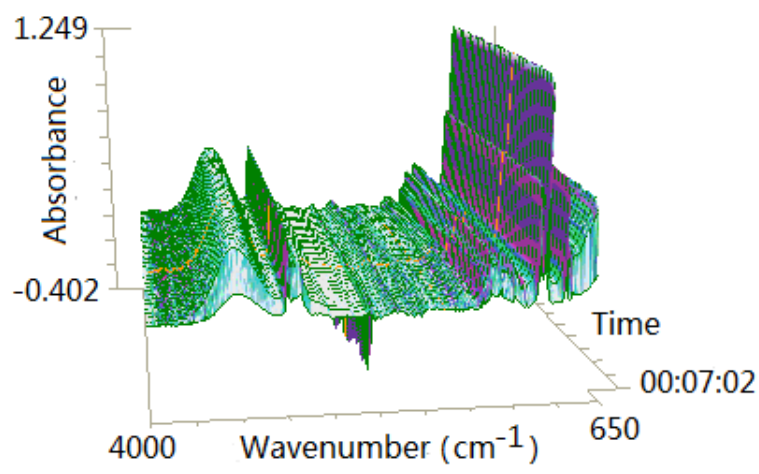

Fig. 1 The 3D online FTIR spectra

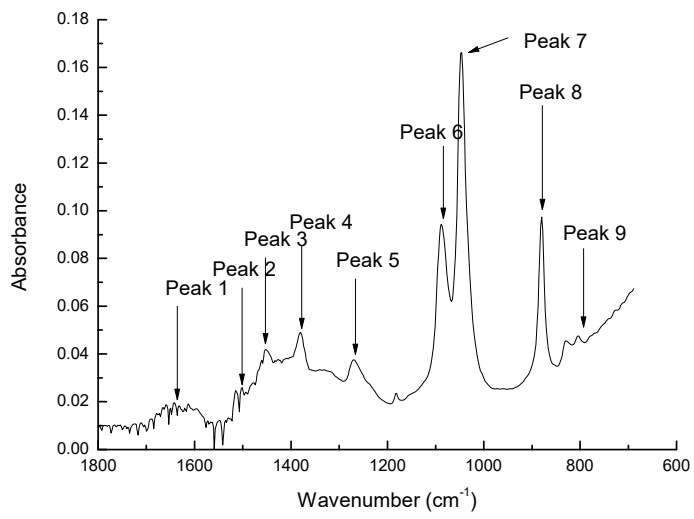

Fig. 3 FTIR of component 2

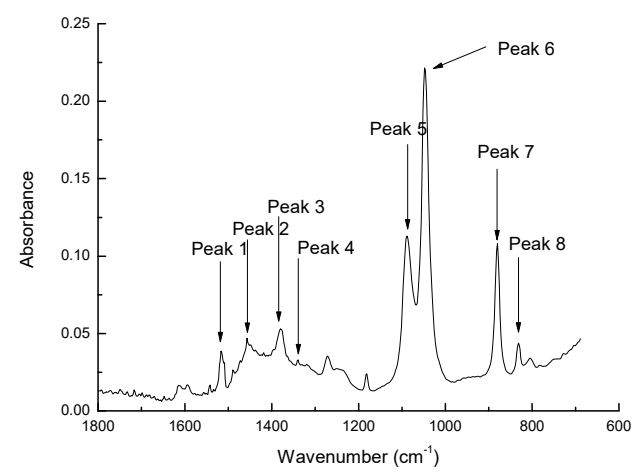

Fig. 5 FTIR of component 4

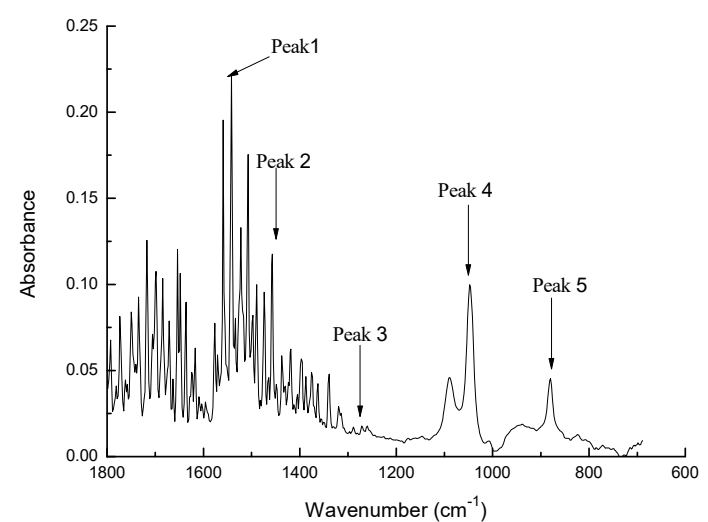

Fig. 2 FTIR of component 1

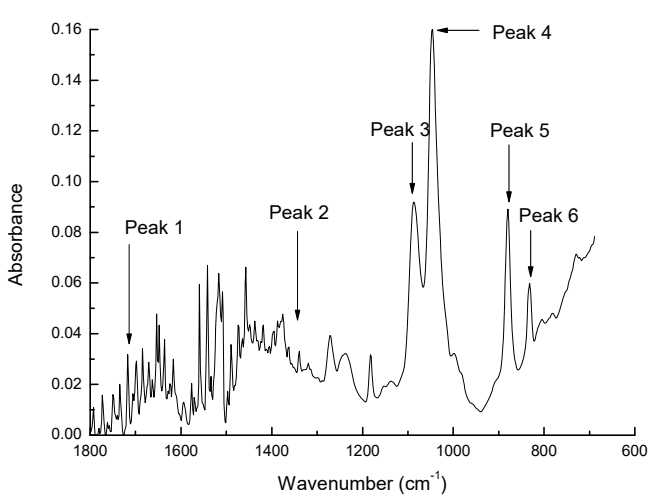

Fig. 4 FTIR of component 3

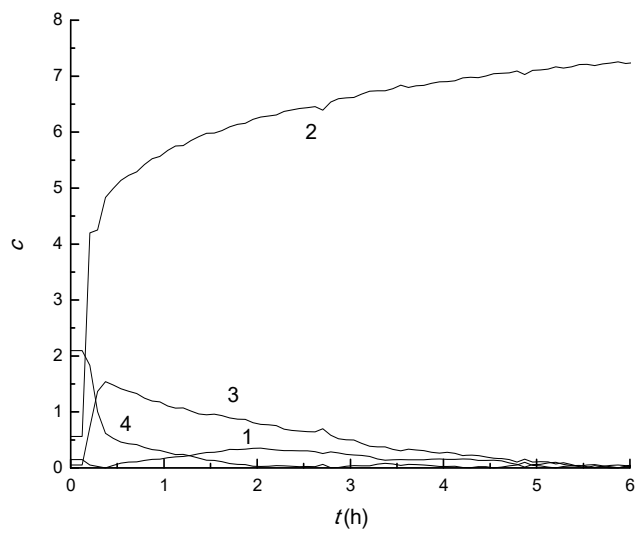

Fig. 6 The relative concentration of component 1-4 versus reaction time

In the FTIR spectrum of component 4 (Fig. 5), the absorption at $1516 \mathrm{~cm}^{-1}$ (peak 1) is attributed to benzene skeleton vibration. The absorption at $1456 \mathrm{~cm}^{-1}$ (peak 2) is attributed to methyl and 
methylene bending vibration. The absorption at $1381 \mathrm{~cm}^{-1}$ (peak 3) is attributed to methyl symmetric bending vibration. The absorption at $1341 \mathrm{~cm}^{-1}$ (peak 4) is attributed to $\mathrm{O}-\mathrm{H}$ plane bending vibration. The absorptions at $1090 \mathrm{~cm}^{-1}$ (peak 5) and $1045 \mathrm{~cm}^{-1}$ (peak 6) are attributed to $\mathrm{C}-\mathrm{O}$ stretching vibration. The absorption at $881 \mathrm{~cm}^{-1}$ (peak 7) is attributed to adjacent C-H bending vibration in benzene ring. The absorption at $837 \mathrm{~cm}^{-1}$ (peak 8) is attributed to C-H out-of-plane bending vibration. Therefore, the spectrum of component 4 obtained by online FTIR agrees with the nonyl phenol's chemical structure.

Figure 6 gives the plot of relative concentrations $(c)$ of component 1-4 with reaction time. As shown in Fig. 6, the relative concentration of component 1 increases slightly at first, then decreases afterwards with reaction time. Therefore, we can confirm that component 1 should be the intermediate as shown in Scheme 2 according to the changing trend of concentration and the online FTIR spectrum in Fig. 2.

The relative concentration (component 2) increases with reaction time. Therefore, we can confirm that component 2 should be the target product to be synthesized according to the changing trend of concentration and the online FTIR spectrum as shown in Fig. 3.

The relative concentration of component 3 increases sharply at the beginning, then decreases gradually with reaction time. Therefore, we can confirm that component 3 should be formaldehyde according to the changing trend of concentration and the online FTIR spectrum in Fig. 4.

The relative concentration of component 4 decreases gradually with reaction time. Therefore, we can confirm that component 4 should be nonyl phenol according to the changing trend of concentration and the online FTIR spectrum in Fig. 5.

Synthesis reaction mechanism. For reaction $(\mathrm{R} 1)$, we presented a Scheme 2 as shown in reactions (R2-R6), based upon the spectrum obtained by online FTIR detection (Fig. 2-5) and the concentration changing trends of the four components in Fig. 6.

The reaction belongs to Mannich reaction. Component 1 of the intermediate is formed by the reaction of diethylenetriamine with formaldehyde (reaction R2). Phenoxy anion is formed by ionization from nonyl phenol in alkaline condition (reaction R3). The phenoxy anion resonates to carbanion (reaction R4). A keto isomer is formed by the reaction of the carbanion with the intermediate (reaction R5). The final asphalt emulsifier product is produced by the keto-enol tautomerism from the keto isomer (reaction R6).<smiles>NCCCCCCCCCNCCNCCNCC(O)NCCNCCN</smiles>

(Component 1: intermediate)

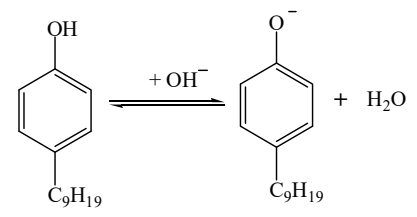

$\overbrace{\mathrm{C}_{9} \mathrm{H}_{19}}^{\mathrm{O}^{-}}$

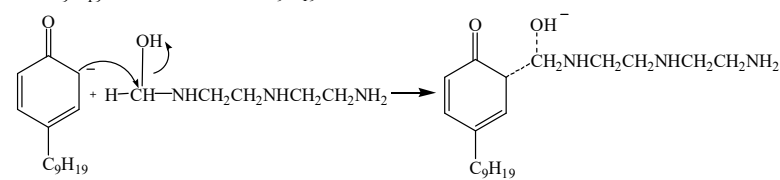

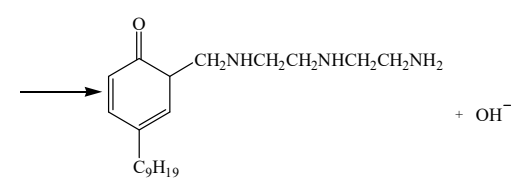




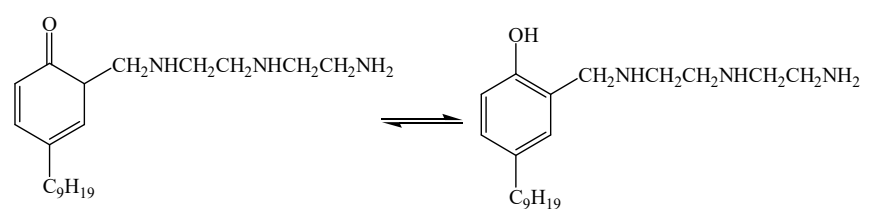

(Component 2: Product)

Scheme 2 Synthesis reaction mechanism

Performance evaluation for the asphalt emulsion. The emulsifier gives excellent emulsifying ability for A-100 asphalt. The prepared asphalt emulsion had higher storage stability. There is not asphalt phase and water phase to be separated after 5 days. The mixing time was $2 \mathrm{~s}$. It indicates that the emulsifier belongs to a rapid-set emulsifier of asphalt. It can be applied to the road pavement construction of tack coat and chip seal.

\section{Conclusion}

A new cationic asphalt emulsifier was synthesized by the reaction of nonyl phenol, diethylenetriamine and formaldehyde. The synthesis process was detected by online FTIR technique and the intermediate was found. Based upon the experimental information, a reaction mechanism was suggested for the reaction. The emulsifier shows excellent emulsifying ability for A-100 asphalt. The emulsifier belongs to rapid-set emulsifier of asphalt.

\section{Acknowledgement}

The authors would like to thank the financial support from the Shandong Provincial Science and Technology Project (No. 2015GGX107005).

\section{References}

[1] H.P. Li, H. Zhao, K. Liao. The preparation of asphalt emulsions with dissymmetric Gemini quaternary ammonium salts cationic surfactants. Energy Sources, Part A, 2013, 35:2285-2293.

[2] L.E. Chávez-Valencia, E. Alonso, A. Manzano, J. Perez, M.E. Contreras, C. Signoret. Improving the compressive strengths of cold-mix asphalt using asphalt emulsion modified by polyvinyl acetate. Construction \& Building Materials, 2007, 21(3):583-589.

[3] G. Barreto, L. Grampre. Bituminous products and aqueous emulsions based on bituminous products and uses thereof. USP 7,951,858 B2, 2011-05-31.

[4] J.M. Wates, A. James. Polybetaines as asphalt emulsifiers. USP 6,540,822 B2, 2003-04-01.

[5] W.T. Zdybak. Anionic bituminous emulsions. USP 4,427,449, 1984-01-24.

[6] M.W. Shuey, R.S. Custer. Polyphenolic vegetable extract/surfactant compositions as universal bitumen/water emulsifiers. USP 5,650,000, 1997-07-22.

[7] A. Jada, C. Florentin, S. Mariotti. Study of the electrical properties of cationic bitumen emulsions by microelectrophoresis. Advances in Colloid and Interface Science, 2004, 108-109:127-132.

[8] P. Schilling. High viscosity cationic slow-set and medium-set emulsions. USP 6,077,888, 2000-06-20.

[9] P.E. Graf, S.J. Agazzi, E.J. Vanderzanden. Open-graded asphalt emulsion mixes. USP 4,985,079, 1991-01-15.

[10] Wu B, Yang Y, Pan X, Wang X, Liu G. Novel synthetic method of lingninamine-type asphalt emulsifier. J. Northeast For. Univ., 1992, 3(1):89-94. 
[11] ø. Rist, A. Rike, L. Ljones, Per H.J. Carlsen. Synthesis of novel diammonium gemini surfactants. Molecules, 2001, 6: 979-987.

[12] G. I. G. Moore, M.S. Terrazas. Fluorinated Gemini surfactants. USP 7,164,041 B1, 2007-01-16.

[13] J.F. Lu, H.G. Wang, D. Liu, K.J. Liao. Synthesis of dissymmetric Gemini quaternary ammonium salts cationic asphalt emulsifiers. Industrial Catalysis, 2006, 14(6): 62-64.(in Chinese)

[14] Velázquez M M, Ortega F, Monroy F, et al. Langmuir monolayers of the zwitterionic surfactant hexadecyl 1-N-L-tryptophan glycerol ether. Journal of Colloid \& Interface Science, 2005, 283(1):144-152.

[15] J.L. Bigorra, N.B. Gilabert, M.O. Hernandez, et al. Formulations for cleaning hard surfaces comprising a betaine surfactant having exactly 12 carbon atoms. USP 6,015,780, 2000-01-18.

[16] Yoshimura T, Ichinokawa T, Kaji M, Esumi K. Synthesis and surface-active properties of sulfobetaine-type zwitterionic gemini surfactants. Colloids and Surfaces A: Physicochem. Eng. Aspects, 2006, 273:208-212.

[17] N. Li, L. Shi, X. Gong, Q. Xu, X. Liu, X. Wang. Synthesis of a novel cationic asphalt emulsifier and its investigation by online FTIR spectrophotometry. Research on Chemical Intermediates, 2015, 41(4): 1935-1950.

[18] L. Shi, M. Sun, N. Li, B. Zhang. A novel betaine type asphalt emulsifier synthesized and investigated by online FTIR spectrophotometric method. Chem. Ind. Chem. Eng. Q., 2015, 21(1):113-121. 